\title{
Poisson Distribution
}

National Cancer Institute

\section{Source}

National Cancer Institute. Poisson Distribution. NCI Thesaurus. Code C53212.

A distribution function used to describe the occurrence of rare events or to describe the sampling distribution of isolated counts in a continuum of time or space. This special probability distribution can apply to the number of discrete independent random events occurring in a given interval when knowing their average rate of occurrence over a very long interval. 